\title{
Water jet streams modeling for firefighting activities with the aid of $\mathrm{CDF}$
}

\author{
F. A. Ponziani ${ }^{1,2} \&$ A. Tinaburri ${ }^{1,2}$ \\ ${ }^{1}$ Central Direction for Prevention and Technical Safety, \\ Department of Firefighters, Ministry of the Interior, Italy \\ ${ }^{2}$ Department of Industrial Engineering, \\ Rome "Tor Vergata" University, Italy
}

\section{Abstract}

The aim of this study is to illustrate the advantage of using computational fluid dynamics (CFD) in order to help manage fire-fighting operations on the field.

The jet streams from a water nozzle in an open field are investigated in terms of essential hydraulics characteristics such as overall shape patterns and throw.

The model developed by CFD is also based on the measures taken in an open field test programme for characterizing the nozzle of a robot kit designed for fire-fighting operations in hostile environments. The observations made during the tests are used in two ways: one way is to try to feed information to the CFD model on the hydraulics in the jet issuing from the nozzle, the other way is to compare the overall outcome from the CFD model to the results obtained in the test programme.

The first part of the study describes the features of the nozzle set up (geometry, positioning, pressures), the open field environment of the tests (space, wind), the outcome observed (stream shape, flow rate, throw). The second part of the study describes the CFD model in terms of specific characteristics of the nozzle (emerging shape patterns), of the numerical domain set (global dimensions and wind influence) and in terms of the outcome obtained (stream shape, flow rate, throw). The comparison between the tests results and the numerical results completes the study and forms the basis for a proposed combined use of information gained from real and associated virtual environment, which can be of help in managing fire-fighting activities.

Keywords: water jet, throw, CFD, droplet diameter, particles injection rate. 


\section{Experimental setup}

The experimental set-up was designed to assess the hydraulics and the functional requirements of the fire-fighting module of a robot-kit intended for use in hostile environments. The campaign of tests was carried on in the Hydraulics Laboratory of the Central Direction for Prevention and Technical Safety - ex Centro Studi Esperienze that is Studies and Experiences Centre - of the Italian National Firefighters [1].

The tests were divided in two groups: one to get information about the flow rates and the pressures at the nozzle, one to get information about the throws and the flow patterns of the water jet.

In order to test the flow rates and the pressures at the nozzle, a properly designed circuit was used inside the laboratory and in the open field where both series and parallel assembly of the hose branches carrying water to the end nozzle were investigated. Various configurations were tested, also varying the type of the water pumps, and several devices were used to monitor the dynamics and obtain the information required (Figure 1).
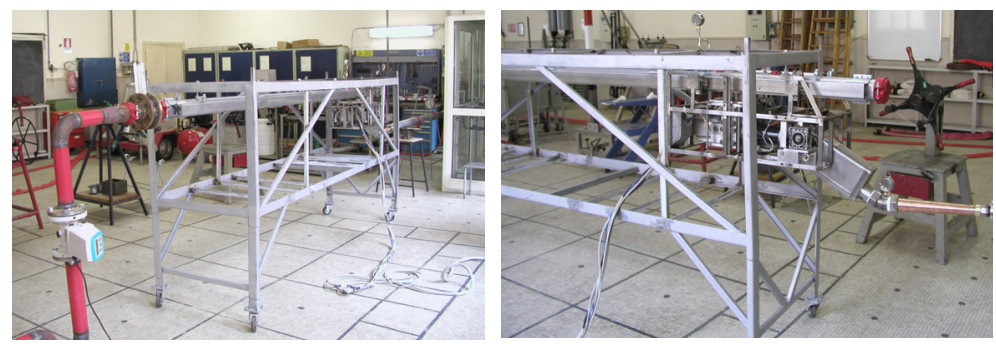

Figure 1: Hydraulic assembly tested in the laboratory.

In order to test the throws and the flow patterns of the water jet, a properly designed configuration was assembled in the open space outside the Lab where different nozzle positions were tested. By varying the hydraulics circuits coming from the Lab, several configuration were tested, and the dynamics and the information required were obtained using several monitoring devices (Figure 2).
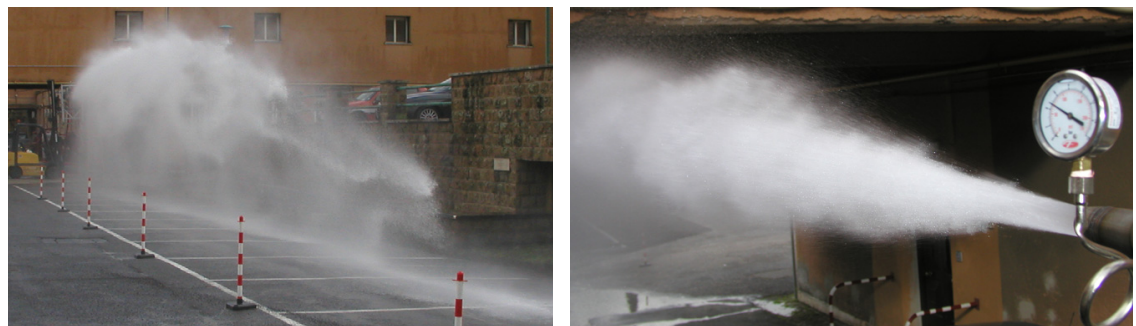

Figure 2: Hydraulic assembly in the open field. 
The nominal flow rate test was made using a properly set prototype of the robot-kit unit, composed of a horizontal DN 80 pipe, with one plug-in valve, and of the monitor assembly mounted on a hand-movable frame. A local panel was used to control the water flowing in the same way as on board the fire-fighting module, thus simulating the operational conditions of the robot-kit unit. The test assembly reproduces the plug-in condition made with the nozzle in the horizontal plane and full water jet (Figure 3).

The flow rate is measured at a reference pressure of $0.6 \mathrm{MPa}( \pm 0.02 \mathrm{MPa})$ at the nozzle (position E in Figure 3). The nozzle (position F) inside diameter was measured with a digital calibre Mitutoyo resulting to be $30 \mathrm{~mm}$. The flow rate test was made through several measures always maintaining an open circuit, having water flowing out from the nozzle. Depending on the circuit prepared, two types of pump stations and subsequent feeding tanks were used: one fire engine pump with a $7.5 \mathrm{~m}^{3}$ tank or one motor pump with a $5 \mathrm{~m}^{3}$ storage tank, having both the required characteristics to maintain the flow in the conditions fixed for each test performed. In each case, a flow measuring section (position B) was provided between the pump and the nozzle unit, on the feeding branch of water for the outflow. The flow rate measurement was made by means of an electromagnetic device Siemens Sitrans F M MAG 5100 W, class II of Directive $2004 / 22 /$ CE "MID" with uncertainty $<0.1 \%$, digital display with scale end $100 \mathrm{~m}^{3} / \mathrm{h}(\sim 1667 \mathrm{l} / \mathrm{min})$. All the tests were made at a constant pressure of $0.6 \mathrm{MPa}$ (6 bar) at the reference position foreseen in the test assembly, controlled by means of a manometer (AF Engineering with scale end 16 bar) at the nozzle entrance (position E). For the $30 \mathrm{~mm}$ nozzle diameter, the average of the measures done resulted in $1251 \mathrm{l} / \mathrm{min}$ at the reference pressure of $0.6 \mathrm{MPa}$ (6 bar).

The effective throw test was made through several measures in the open field, varying the height of the installation so to have the nozzle axis placed at the heights of $2 \mathrm{~m}, 3 \mathrm{~m}$ and $4 \mathrm{~m}$ above the ground level according to the system intended use. The test assembly reproduces the plug-in condition in the installed system, with the nozzle in the horizontal plane and full water jet (Figure 4).

All the tests were performed at the same reference pressure of $0.6 \mathrm{MPa}$ (position E in Figure 6) and with the same nozzle configuration (30 $\mathrm{mm}$ internal diameter).

The effective throw measurements at the various heights of installation were made through the analysis of the video recordings of the tests, examining the impact area of the water jet on the ground in conjunction with the distance from the outflow nozzle and using a marking mesh. The measurements were done in calm wind conditions (Beaufort scale: grade 0 , wind 0 to $0.5 \mathrm{~m} / \mathrm{s}$, corresponding to a smoke plume that rises vertically) and the results are reported in Table 1.

Table 1: Effective throw measurements as a function of height.

\begin{tabular}{|l|c|c|c|}
\hline Installation height $h(\mathrm{~m})$ & 2 & 3 & 4 \\
\hline Effective throw $l^{\circ} \mathrm{eff}(\mathrm{m})$ & 21 & 24 & 27 \\
\hline
\end{tabular}

Reference pressure: $0.6 \mathrm{MPa}$ - nozzle ID $30 \mathrm{~mm}-\alpha=0^{\circ}$ - full water jet. 

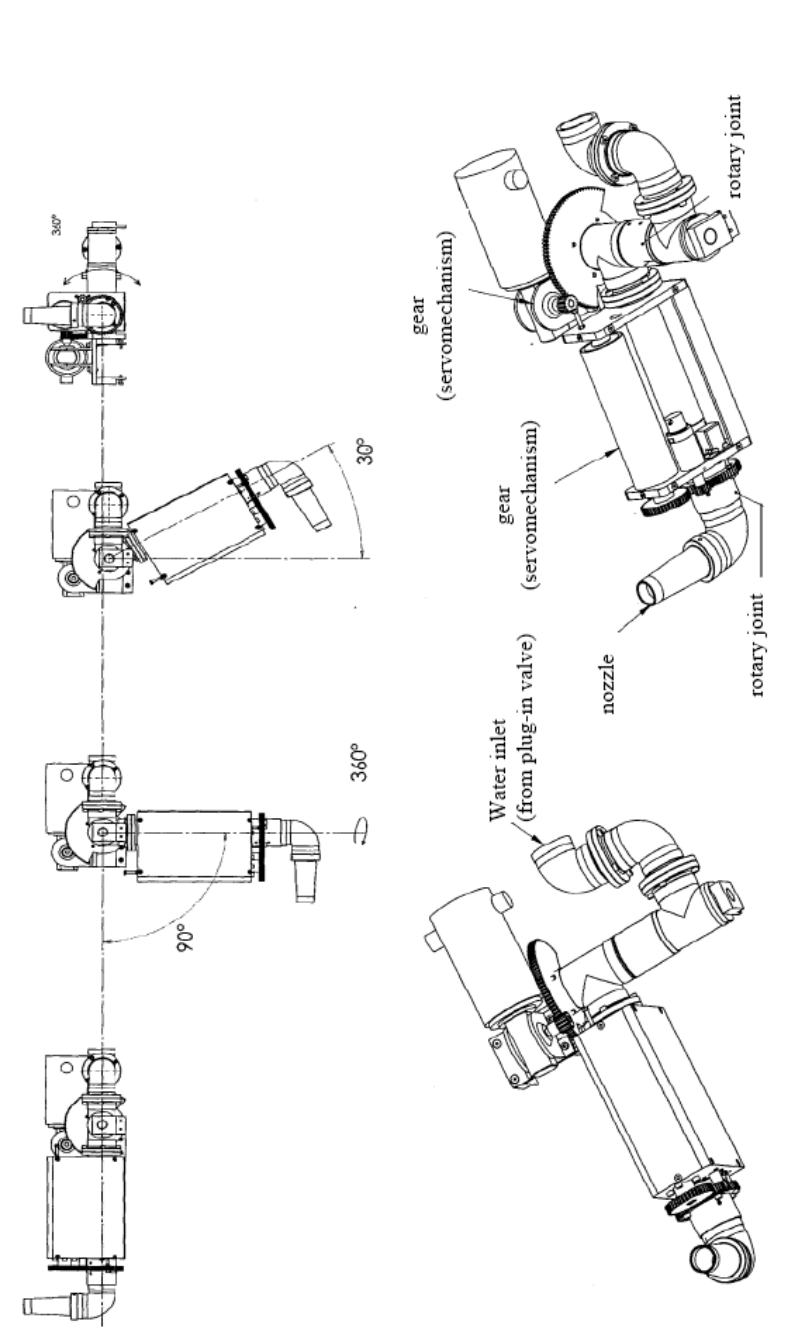

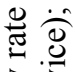

定

$\ddot{\varphi} \stackrel{800}{=}$

产

\&

0

कृ

导

○्ن

o

$\stackrel{\frac{\pi}{\pi}}{>}$

ㄹ.

产

30

on

응

巴马

近

르유.

离

ह

을 đ

응

옳

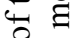

ض̈

㐘

ส

急

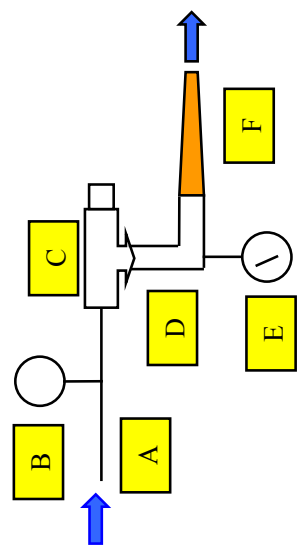

.000

픔.

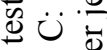

过

음

밍

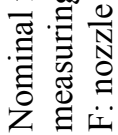

$\ddot{\ddot{\nu}}$ 


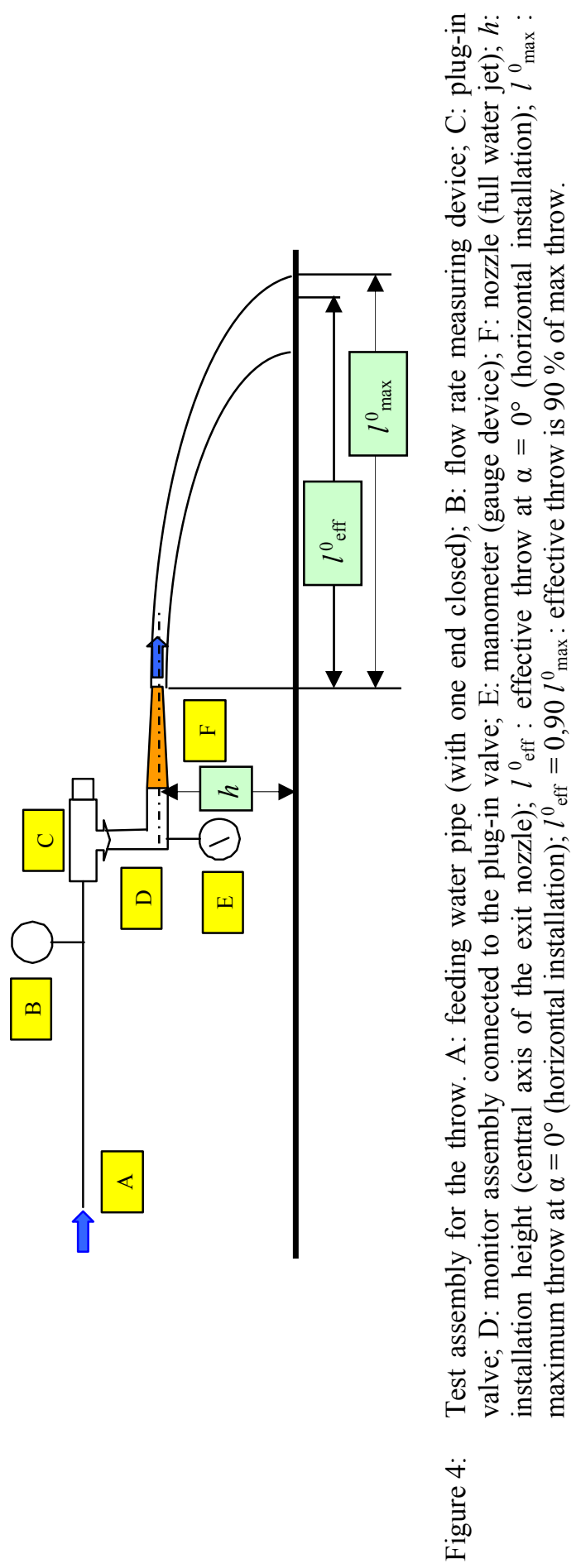


A slight variation in the angle of inclination (say up to about $5^{\circ}$ ) might be considered in the CFD simulations of the effective throw to reproduce the limited misalignment produced by the vibrations generated by the water flow under the conditions tested in the open field (Figure 5).

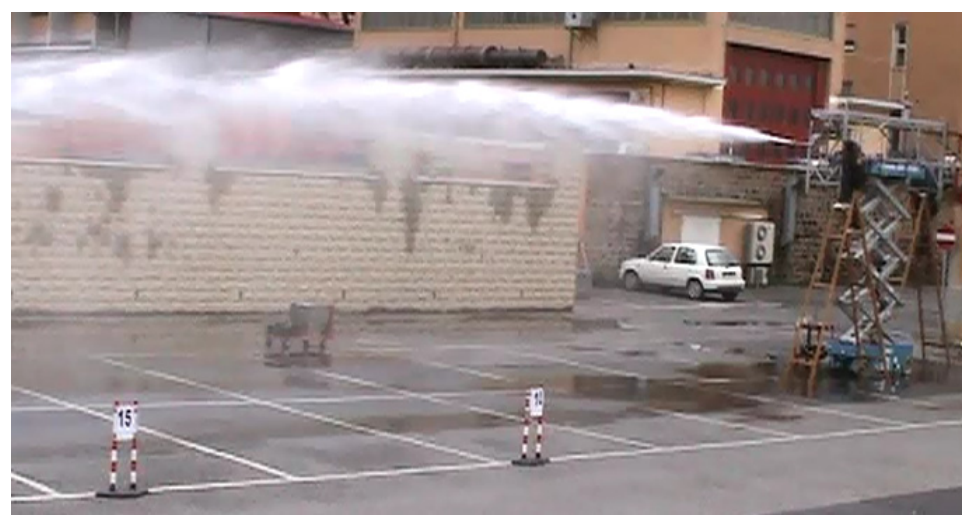

Figure 5: Test assembly in a throw test.

\section{Numerical simulation}

The numerical simulation consisted in a study of the overall water jet properties issuing from the nozzle of the robot kit unit, using the CFD package Fire Dynamics Simulator [2, 3] and Smokeview [4, 5] version 6. Starting from the data obtained in the open field tests, the numerical model was prepared by defining a proper domain and setting the initial conditions for the nozzle water flow. The computational domain represents part of the open field where the tests were conducted, describing an outdoor domain around the water jet stream of rectangular form with overall dimensions: $8 \mathrm{~m}$ width by $40 \mathrm{~m}$ length by $8 \mathrm{~m}$ height, with one bottom boundary as the ground floor and the remaining other external boundaries open to the atmosphere. Multiple meshes are used, and the variation of the mesh grid from coarser to finer is a significant part of the assessment. Since the CFD study refers to the experimental set-up in the open field with the nozzle set at the height of $4 \mathrm{~m}$ above ground level, this same position has been chosen for the nozzle from which a water flow is set to be launched in the numerical simulation. From the hydraulics data obtained in the tests come a flow rate of $1250 \mathrm{1} / \mathrm{min}$ at the pressure of 6 bar issuing from a nozzle with a diameter of $30 \mathrm{~mm}$ : hence an exit velocity of $29.5 \mathrm{~m} / \mathrm{s}$.

Visual observations from the videos and the photographs reveal a conical spray starting with a narrow pattern emerging from the nozzle, whose inclination is slightly above the horizontal. After some checking, the following values are used to initialize the hydrodynamics simulation: $30 \mathrm{~m} / \mathrm{s}$ of exit velocity for the water jet from the nozzle, spray pattern of $12^{\circ}$ issuing from the nozzle, $5^{\circ}$ upward of inclination for the nozzle. 
The practical absence of wind during the open field tests programme has some direct consequences for the CFD runs programme:

- there is no need to accurately investigate the wind scheme that might be used in the numerical simulation;

- there may be expected no significant interference on the water jet stream pattern;

- the high momentum of the water jet relative to the surrounding air is strong enough to maintain the original shape for a long time.

One more important parameter is required to initialize the runs: the median volumetric droplet diameter. This parameter is part of a statistical description of the water flow pattern, and helps to distinguish between different possible characteristics and behaviors [6-8]: finer diameters (on the order of magnitude $100 \mu \mathrm{m})$ are useful to rapidly absorb a vast amount of heat but may be more easily deviated by the thermal and fluid dynamics currents before they reach the target, coarser diameters (on the order of magnitude $1000 \mu \mathrm{m}$ ) are useful to penetrate at a distance reaching the heated target more efficiently but are less effective in rapidly absorbing heat in that they have a less specific surface to exchange heat with the surroundings. Detailed measurements are required to get the values of the droplet diameters issuing from the various nozzles under the various flow configurations. For the aim of this study, the water streams of interest are those able to flow and penetrate at a distance, so the droplet diameter is in the upper range above mentioned. In the absence of further information about the droplet diameters, a sensitivity analysis is conducted and the outcomes are compared to assess the numerical simulations relative to the experimental tests. Three values of the median volumetric droplet diameter are chosen for the numerical initializations: $500 \mu \mathrm{m}, 1000 \mu \mathrm{m}, 2000 \mu \mathrm{m}$. The statistical size droplet distribution and injection are left as the default given by the CFD package (Rosin-Rammler-Lognormal, 5000 particles per second injection).

Due to the current limitation in describing the detailed forms of the finer elements of the water jet streams (knowledge of measured data, interpretations of sub-grid scales ...) one practical approach in modeling for this study is to extract information about global aspects, such as visual shapes and distances travelled by the water stream, and compare the numerical data with the data obtained in the physical tests. To this end, close attention is required for defining the numerical mesh grid, in that even an overall shape of the water stream can sensibly differ from the one observed in the tests. Starting with the $500 \mu \mathrm{m}$ droplet runs, a sensitivity study about the mesh grid of the domain revealed how a finer mesh is required to obtain an overall shape of the water streams that better resembles the one observed in the open tests. As a matter of fact, for the configuration investigated, the change from a $40 \mathrm{~cm}$ cubic cell to a $20 \mathrm{~cm}$ cubic cell has been highly significant (Figure 6).

Studies on finer mesh grids have been done for the $1000 \mu \mathrm{m}$ and $2000 \mu \mathrm{m}$ droplet runs, too. The case of the $1000 \mu \mathrm{m}$ droplets runs, for example, shows an improvement in further refining the mesh, yet in general less marked when the 


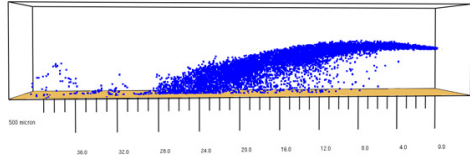

$40 \mathrm{~cm}$ cubic cell mesh

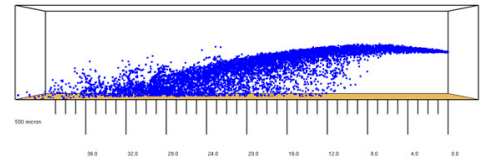

$20 \mathrm{~cm}$ cubic cell mesh

Figure 6: CFD throw and mesh grids for $500 \mu \mathrm{m}$ droplets.

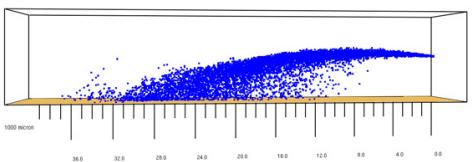

$20 \mathrm{~cm}$ cubic cell mesh

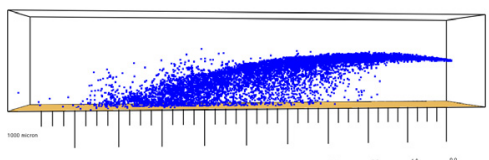

$10 \mathrm{~cm}$ cubic cell mesh

Figure 7: $\quad$ CFD throw and mesh grids for $1000 \mu \mathrm{m}$ droplets.

refinement is from $20 \mathrm{~cm}$ to $10 \mathrm{~cm}$ compared to the $40 \mathrm{~cm}$ to $20 \mathrm{~cm}$ change (Figure 7).

Another parameter to be defined in the modeling is the number of particles injected per second by the nozzle: this serves to refine the simulation, but is not directly related to the very number of water droplets issuing from the nozzle in a real water jet stream. In the absence of sophisticated and expensive measurements, if available, a sensitivity analysis of this parameter may help in evaluating the quality of the numerical simulation and the grade of rendering.

The case of $500 \mu \mathrm{m}$ droplet has been investigated to see the variation in the rendering between 5000 and 20000 particles per second injected from the nozzle. Given the capability of this parameter and the goal of this study, there has not been such a marked improvement as the one obtained refining the mesh grid (Figure 8).

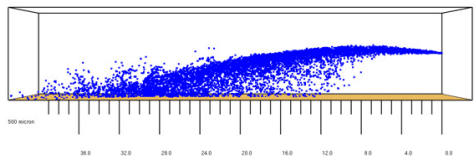

5000 particles per second

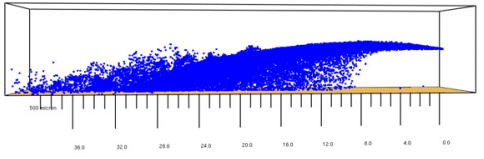

20000 particles per second

Figure 8: CFD throw with a fine mesh grid (20 cm cubic cells) for $500 \mu \mathrm{m}$ water droplets with two differing particles injection rates.

The results show a qualitative agreement with the overall shapes and the pattern observed in the open field tests, with some differences in the throws depending on the droplet dimension chosen for initializing the run (Figures 9 to 11$)$. 

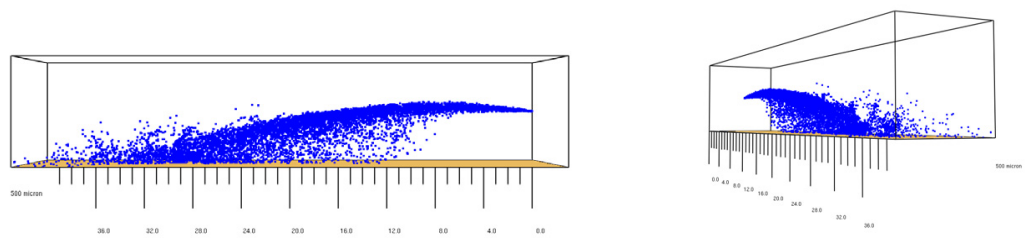

Figure 9: CFD side view and perspective for $500 \mu \mathrm{m}$ droplets and $20 \mathrm{~cm}$ cell size.
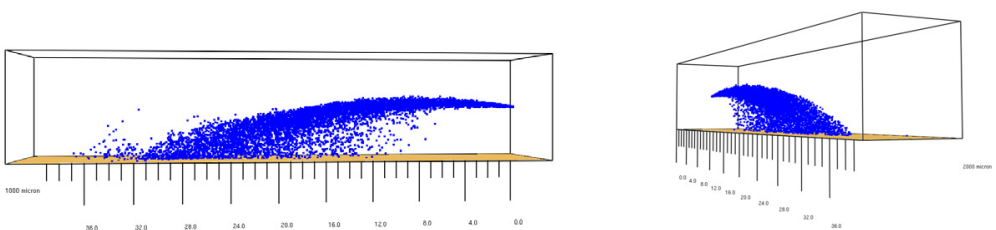

Figure 10: CFD side view and perspective for $1000 \mu \mathrm{m}$ droplets and $20 \mathrm{~cm}$ cell size.
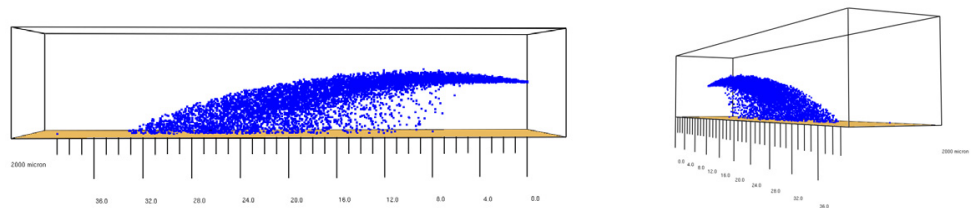

Figure 11: CFD side view and perspective for $2000 \mu \mathrm{m}$ droplets and $20 \mathrm{~cm}$ cell size.

By looking at the global aspects, the elongated form of the stream and the cascading effect to the end pattern appear in the numerical simulations, even if some details cannot be replicated in terms of pulses of water sheets and very fine fringes.

As far as the throw is concerned, the length of about $27 \mathrm{~m}$ of the effective throw obtained in the open field tests is reasonably simulated by the numerical runs, where the global impact on the ground is a bit different depending on the droplet dimension. Taking into account the maximum throw of about $30 \mathrm{~m}$, from which the effective throw is defined as its $90 \%$, it is possible to make further observations (Figure 12).

The global character of the throw is captured in its essential geometry (shape and pattern) and dynamics (distance and impact). Depending on the hydrodynamical parameters used for initializing the runs, there may be more or less fading in the side ends of the water toward the ground, and some mesh 
refinement may add further accuracy in the similarities between the CFD models and the data obtained by the open field tests. Anyway, due to the inherent type of modeling available in the CFD for the water stream, there is not an ever increasing improvement in reducing the grid cell independently of the simulation scope, and an engineering judgement is crucial in order to acquire balanced and useful information.

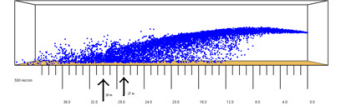

$500 \mu \mathrm{m}$

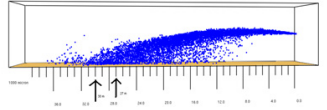

$1000 \mu \mathrm{m}$

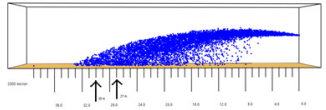

$2000 \mu \mathrm{m}$

Figure 12: CFD comparison about max throw (longer arrow) and effective throw (shorter arrow) at droplets diameter: 500, 1000, $2000 \mu \mathrm{m}$, on a suitable same mesh grid: $20 \mathrm{~cm}$ cell size.

The case of $500 \mu \mathrm{m}$ droplet runs with 5000 particles per second injection is shown as an example. By means of the available capabilities of the numerical modeling, comparison of the results show that the runs with a $20 \mathrm{~cm}$ cubic cell mesh grid and $500 \mu \mathrm{m}$ droplets - ceteris paribus - seem to sufficiently resemble the overall hydraulic characteristics experienced in the open field tests, especially in terms of launching shape and throw pattern (Figure 13).
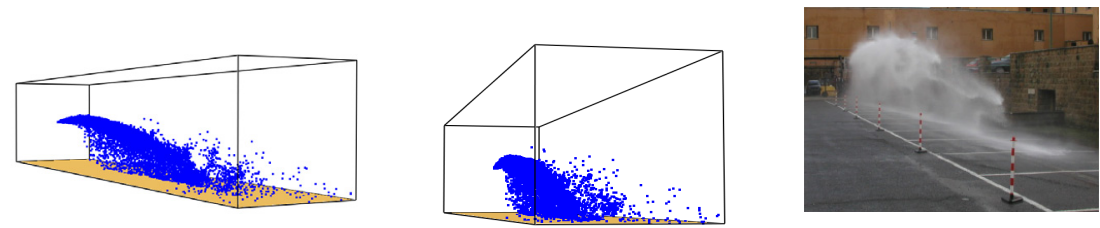

Figure 13: CFD simulation (500 $\mu \mathrm{m}$ droplets diameter, $20 \mathrm{~cm}$ grid cell) vs. test.

On the other hand, when dealing with a fire simulation, a mesh refinement is a necessary part of the study that deserves further considerations. In that case, together with the overall characteristics there are also some more detailed features that are of importance, principally regarding the smoke and flame treatment. In order to understand the order of magnitude involved, there are two important parameters that may be invoked that give information about the fire behavior and about the CFD package resolution of the fire plume.

The former is the Zukoski number, $Z u$, defined as a nondimensional combination of fire and ambient characteristics [6]:

$$
Z u=Q \rho_{a}^{-1} c_{p}^{-1} T_{a}^{-1} g^{-1 / 2} D^{-5 / 2}
$$


where $Q$ is the firepower [MW], $\rho_{a}$ is the ambient air density $\left[\mathrm{kg} \mathrm{m}^{-3}\right], c_{p}$ is the air heat capacity at constant pressure $\left[\mathrm{kg} \mathrm{kJ}^{-1} \mathrm{~K}^{-1}\right], T_{a}$ is the ambient air absolute temperature $[\mathrm{K}], g$ is the acceleration due to gravity $\left[\mathrm{m} \mathrm{s}^{-2}\right], D$ is the fire diameter $[\mathrm{m}]$.

The second is the Plume resolution index PriFDS, defined as a nondimensional combination of fire, ambient and grid cell characteristics [3]:

$$
\operatorname{PriFDS}=\left(Q \rho_{a}^{-1} c_{p}^{-1} T_{a}^{-1} g^{-1 / 2}\right)^{2 / 5} \delta_{x}^{-1}
$$

where in addition $\delta_{\mathrm{x}}$ is the grid cell side [m].

Considering a $4 \mathrm{MW}$ fire from a $4 \mathrm{~m}^{2}$ area in a $10 \mathrm{~cm}$ grid cell side domain at the ambient temperature of $15^{\circ} \mathrm{C}$, the two parameters are:

$$
Z u=0.47 \quad \text { PriFDS }=16.70
$$

showing a suitable accuracy for the fire simulation.

This mesh may now be used to model the adding of the water stream and assess the combined effect of water and fire streams. Showing the example of the $500 \mu \mathrm{m}$ droplet, with 5000 particles per second injection, the mesh refinement from $20 \mathrm{~cm}$ to $10 \mathrm{~cm}$ cubic cells brings about an upgrading of the simulation both in terms of dynamical resolution and visual rendering (Figure 14).
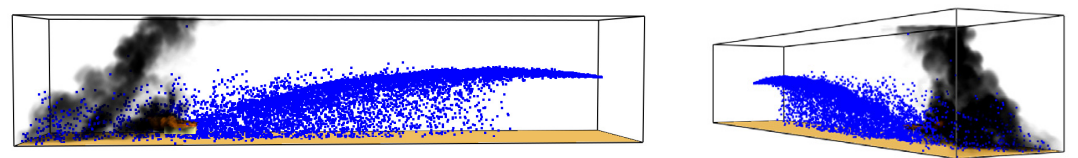

Figure 14: CFD simulation of the interaction of a water jet $(500 \mu \mathrm{m}$ droplets diameter) with a $4 \mathrm{MW}$ fire source, using a $10 \mathrm{~cm}$ cubic cell mesh.

The essentials aspects are captured in the overall dynamics: the water stream flows and arrives on the fire target; the flame is shortened and bended; the smoke is pushed and deflected. Some finer details are also captured: the cascading of the water stream toward the fire, the twisting smoke curls rising. As for the smoke plume deflection caused by the water flow interaction with the fire source, further investigation is needed to deepen the understanding of this particular flow field and to assess the real occurrence in a field test.

\section{Conclusion}

The aim of this study was to illustrate how CFD may be an advantageous tool to help manage fire-fighting matters. As for the CFD water jet stream of this study, several results have been obtained by combining physical and numerical information: proper comparisons are set (influence of water droplets model), 
some choices appear better than others (suitable mesh grids), relative positions may be assessed (water stream on fire target). By a combined use of information acquired through numerical simulation and physical tests, it is possible to schedule a virtual environment where to compare and assess different strategies.

These strategies may represent, for example, the setting-up of a specific test or the outcome of various operations. Always bearing in mind the limitations of such an approach, principally related to a general lack of precise data to rely upon and to the inherent cut-off of the virtual modeling, the possibility of doing several runs may be nevertheless of practical importance. In fact, the virtual data that are processed may be examined and compared, so that at least some of the characteristic parameters involved may be found out, and various strategies may be examined, compared or discarded.

\section{References}

[1] Tinaburri, A. \& Ponziani, F.A., CUAP 11.03/06 Fire-fighting robot kit to be used in tunnels, subsurface facilities and restricted spaces, European Organization for Technical Approvals, Bruxelles, 2012.

[2] Fire Dynamics Simulator (Version 6) - Technical Reference Guide; NIST Special Publication 1018, 2014, Online. http://www.nist.gov/el/fire research/fds smokeview.cfm.

[3] Fire Dynamics Simulator (Version 6) - User's Guide; NIST Special Publication 1019, 2014, Online. http://www.nist.gov/el/fire research /fds smokeview.cfm.

[4] Smokeview (Version 6) - Technical Reference Guide, NIST Special Publication 1017-2, 2014, Online. http://www.nist.gov/el/fire research /fds_smokeview.cfm.

[5] Smokeview (Version 6) - User's Guide, NIST Special Publication 1017-1, 2014, Online. http://www.nist.gov/el/fire_research/fds_smokeview.cfm.

[6] Di Nenno, P.J., The SFPE Handbook of Fire Protection Engineering, NFPA, pp. 4-311, 4-319, 2002.

[7] Fire-fighting flow rate; London Fire Brigade, Grimwood, 2005 www.firetactics.com.

[8] Vaari J., Kling T., Hostikka S., Rinne T. \& Ketola J., Simulating the Rescue Service Response in a Railway Tanker Fire, Proc. of the $13^{\text {th }}$ Int. Conf. Interflam, Interscience, pp. 1359-1369, 2013. 